\title{
Role of Hydroxyl Species in Hydrogen Oxidation Mechanism: a DFT Study
}

\author{
Supporting Information
}

Zhiping Feng, Li Li*, Xingqun Zheng, Jing Li, Na Yang, Wei Ding*, Zidong Wei* The State Key Laboratory of Power Transmission Equipment \& System Security and New Technology, Chongqing Key Laboratory of Chemical Process for Clean Energy and Resource Utilization, Chongqing Key Laboratory of Theoretical and Computational Chemistry, School of Chemistry and Chemical Engineering, Chongqing University, Shazhengjie 174, Chongqing 400044, P. R. China

*E-mail: liliracial@cqu.edu.cn (L.L.);
**E-mail: $\underline{\text { dingwei128@,cqu.edu.cn (W.D.); }}$
***E-mail: zdwei@cqu.edu.cn (Wei),Tel: +86 2365678945 (Z.W.) 
Table S1 Adsorption energy of each site on Pt(110)

\begin{tabular}{ccccc}
\hline$\Delta \mathrm{E}(\mathrm{eV})$ & $\boldsymbol{t}$ site & $\boldsymbol{s}$ - $\boldsymbol{b}$ site & $\boldsymbol{l}$ - site & hollow site \\
\hline $\mathrm{H}_{2} \mathrm{O}^{*}$ & -0.469 & change to $\boldsymbol{t}$ site & change to $\boldsymbol{t}$ site & change to $\boldsymbol{t}$ site \\
$\mathrm{H}^{*}$ & -2.870 & -2.888 & -2.491 & change to $\boldsymbol{t}$ site \\
$\mathrm{OH}^{*}$ & -3.394 & -3.507 & -3.378 & change to $\boldsymbol{t}$ site \\
\hline
\end{tabular}

Note: The energies of sole $\mathrm{H}, \mathrm{H}_{2} \mathrm{O}$ and $\mathrm{OH}$ are as the standard for computation

Table S2 Relevant thermodynamic data of species ${ }^{1}$

\begin{tabular}{lllll}
\hline & $E_{D F T}$ & ZPE & $T \triangle S_{\exp }$ & ZPE-T $\triangle S$ \\
\hline $\mathrm{H}_{2} \mathrm{O}(\mathrm{g})$ & -14.226 & 0.560 & $0.67(0.035$ bar $)$ & -0.110 \\
& & & & \\
$\mathrm{H}_{2}(\mathrm{~g})$ & -6.771 & 0.270 & 0.403 & -0.060 \\
\hline
\end{tabular}

Note: The entropy of $\mathrm{H}$ at standard conditions: $\mathrm{T}=298 \mathrm{~K}$ and $\mathrm{P}=1$ bar, while the value for $\mathrm{H}_{2} \mathrm{O}$ is at $\mathrm{T}=298 \mathrm{~K}$ and $\mathrm{P}=0.035$ bar, when the Gibbs free energy of $\mathrm{H}_{2} \mathrm{O}(\mathrm{l})$ is equal to that of $\mathrm{H}_{2} \mathrm{O}(\mathrm{g})$. The calculated DFT total energies are for $\mathrm{H}_{2}$ and $\mathrm{H}_{2} \mathrm{O}$ in gas phase. All the energies are in $\mathrm{eV}$.

Table S3 The oxidation potential of metals $\left(\mathrm{U}_{\text {oxidation }}\right)^{2}$

\begin{tabular}{cccccc}
\hline System & $\mathrm{Pt}(\mathrm{OH})_{2}$ & $\mathrm{Ir}_{2} \mathrm{O}_{3}$ & $\mathrm{Pd}(\mathrm{OH})_{2}$ & $\mathrm{Ni}(\mathrm{OH})_{2}$ & $\begin{array}{c}\mathrm{Pt}(\mathrm{OH})_{2} \\
(\mathrm{PtRu}(110))\end{array}$ \\
\hline $\mathrm{U}_{\text {oxidation }}$ & 0.140 & 0.098 & 0.070 & -0.720 & 0.140 \\
\hline
\end{tabular}

Note: The $\mathrm{U}_{\text {oxidation }}$ of $\mathrm{Pt}$ represents the oxidation potential of $\mathrm{PtRu}(110)$ because the $\mathrm{Pt}$ atoms on PtRu(110) are the active sites for HOR/HER. 
Table S4 The bonding length and -ICOHP values of $\mathrm{Pt}-\mathrm{O}\left(\mathrm{H}_{2} \mathrm{O}^{*}\right)$ on $\mathrm{Pt}(110)$ with and without $\mathrm{OH}^{*}$

\begin{tabular}{ccc}
\hline \multirow{2}{*}{ System } & \multicolumn{2}{c}{ Pt-O(H2O*) bond } \\
& $\mathrm{d}_{\mathrm{Pt}-\mathrm{O}} / \AA$ & $-\mathrm{ICOHP}(\mathrm{Pt}-\mathrm{O}) / \mathrm{e}$ \\
\hline $\mathrm{Pt}$ & 2.267 & 2.708 \\
$\mathrm{Pt}-\mathrm{OH}^{*}$ & 2.137 & 3.706 \\
$\mathrm{Pt}-2 \mathrm{OH}^{*}$ & 2.157 & 3.514 \\
\hline
\end{tabular}

(a)

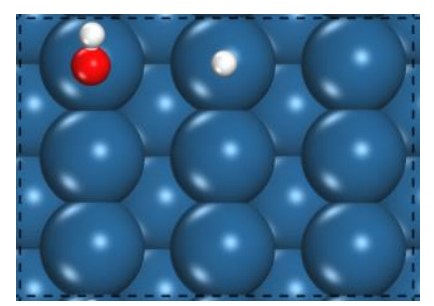

$\mathrm{d}_{\mathrm{Pt}-\mathrm{H}}=1.569 \AA$

$\Delta \mathrm{E}_{\mathrm{H}^{*}}=-0.591 \mathrm{eV}$ (b)

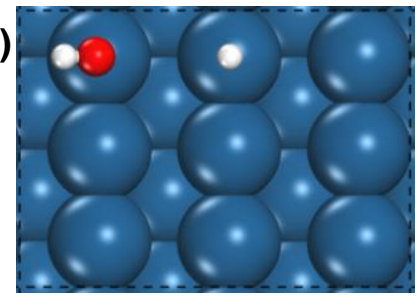

$$
\begin{aligned}
\mathrm{d}_{\mathrm{Pt}-\mathrm{H}} & =1.569 \AA \\
\Delta \mathrm{E}_{\mathrm{H}^{*}} & =-0.591 \mathrm{eV}
\end{aligned}
$$

(c)

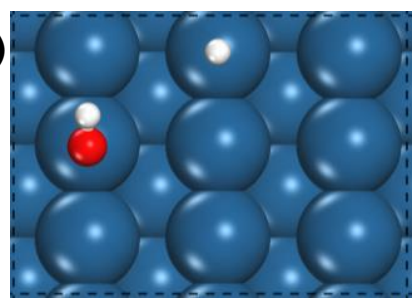

$$
\begin{aligned}
\mathrm{d}_{\mathrm{Pt}-\mathrm{H}} & =1.569 \AA \\
\Delta \mathbf{E}_{\mathrm{H}^{*}} & =-0.599 \mathrm{eV}
\end{aligned}
$$

Figure $\mathbf{S} 1 \mathrm{H}^{*}$ adsorption with far- $\mathrm{OH}^{*}$

(a)

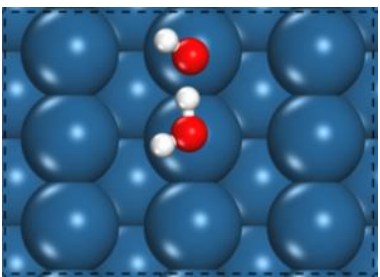

$\mathrm{d}_{\mathrm{Pt}-0}=2.137 \AA$

$\Delta \mathbf{E}_{\mathrm{H}_{2} \mathrm{O}^{*}=-1.055 \mathrm{eV}}$

(c)

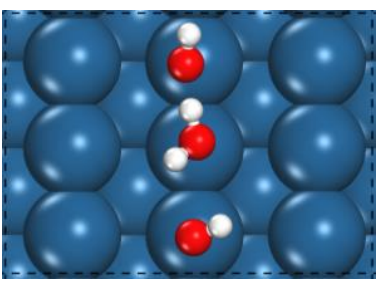

$\mathrm{d}_{\mathrm{Pt}-0}=2.157 \AA$

$\Delta \mathrm{E}_{\mathrm{H}_{2} \mathrm{0}^{*}=-1.406 \mathrm{eV}}$

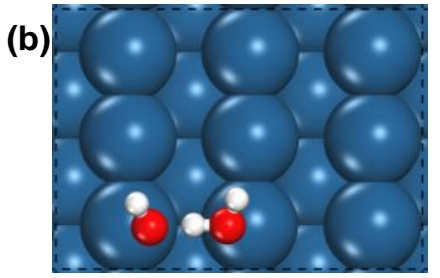

$\mathrm{d}_{\mathrm{Pt}-0}=2.149 \AA$

$\Delta \mathrm{E}_{\mathrm{H}_{2} \mathrm{O}^{*}}=-0.983 \mathrm{eV}$

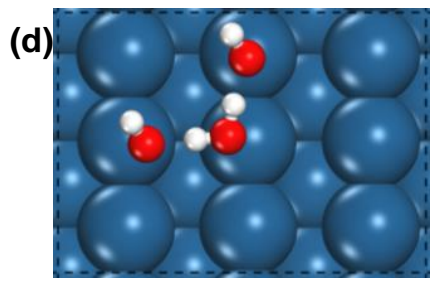

$\mathrm{d}_{\mathrm{Pt}-0}=2.119 \AA$

$\Delta \mathbf{E}_{\mathrm{H}_{2} \mathbf{O}^{*}}=-1.260 \mathrm{eV}$

Figure S2 The comparison for the adsorbed $\mathrm{H}_{2} \mathrm{O}$ between the surfaces with far- $\mathrm{OH}^{*}$ and near-OH* 


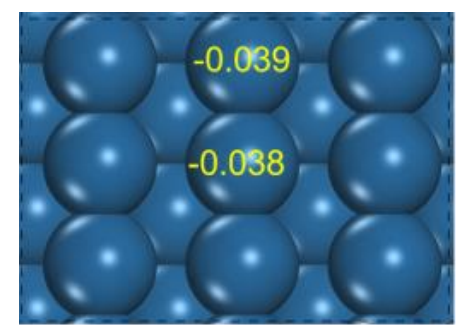

$\operatorname{Pt}(110)$

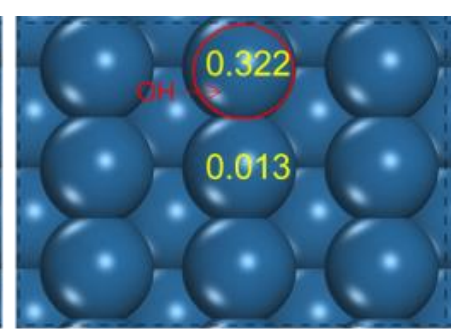

$\mathrm{Pt}(110)-\mathrm{OH}^{*}$

Figure S3 Bader charge of $\mathrm{Pt}$ atoms on $\mathrm{Pt}(110)$ and $\mathrm{Pt}(110)-\mathrm{OH}^{*}$ surface

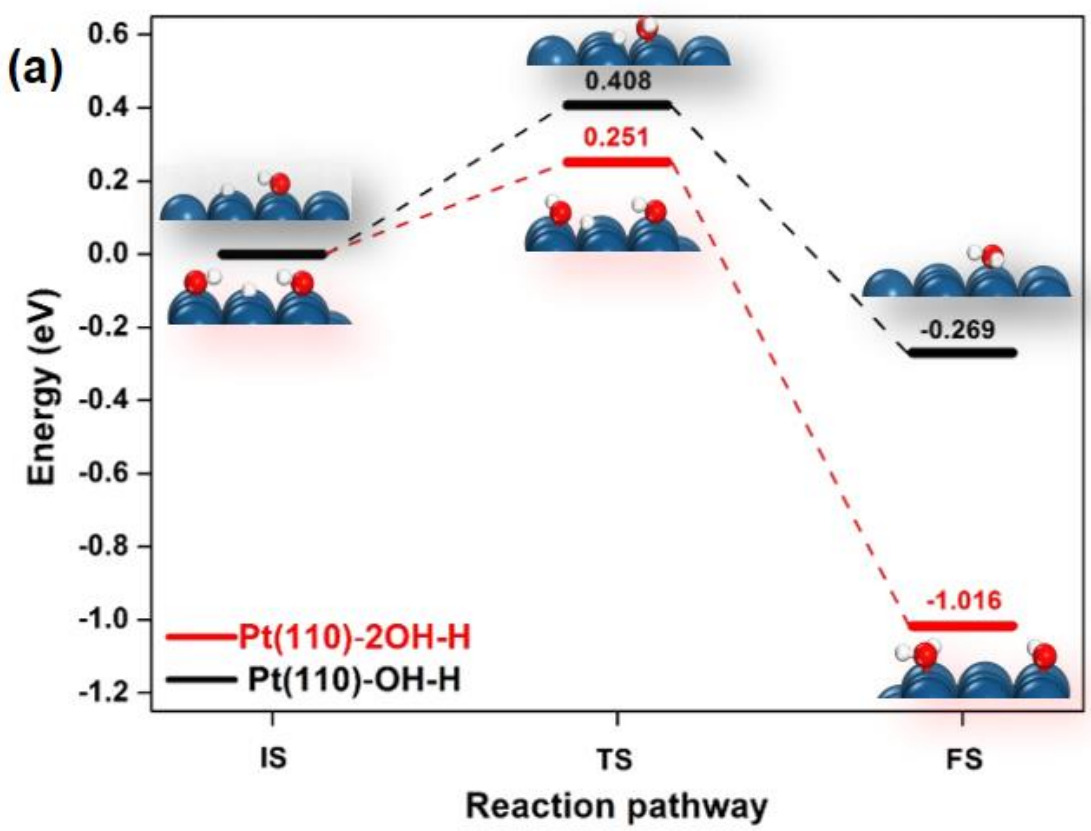

Figure S4 Energy path for $\mathrm{H}^{*}+\mathrm{OH}^{*}=\mathrm{H}_{2} \mathrm{O}^{*}$ on $\mathrm{Pt}(110)$ surface with and without $\mathrm{OH}^{*}$. The insets are the side view of the initial state (IS), transitional state (TS) and final state (FS);
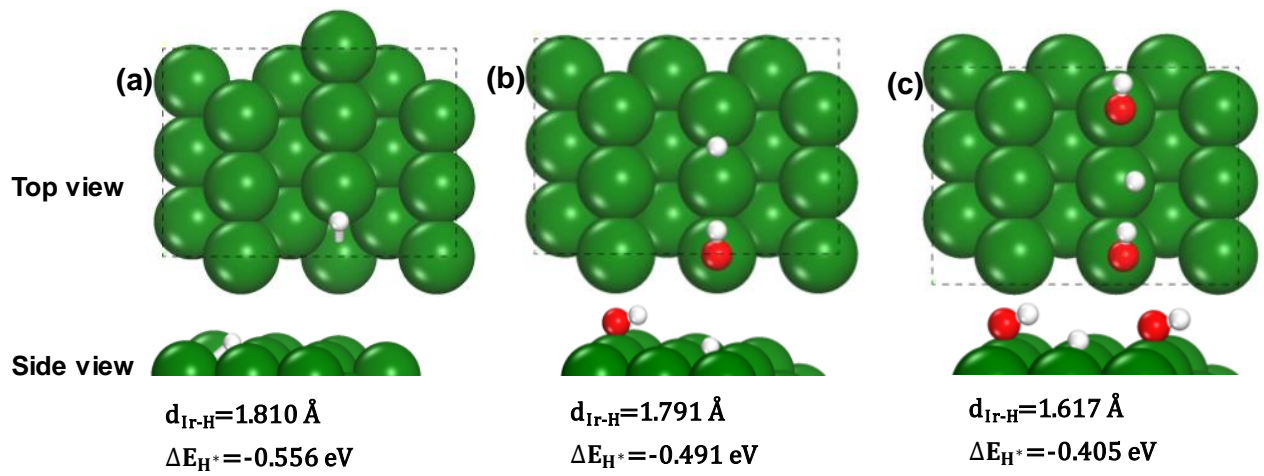

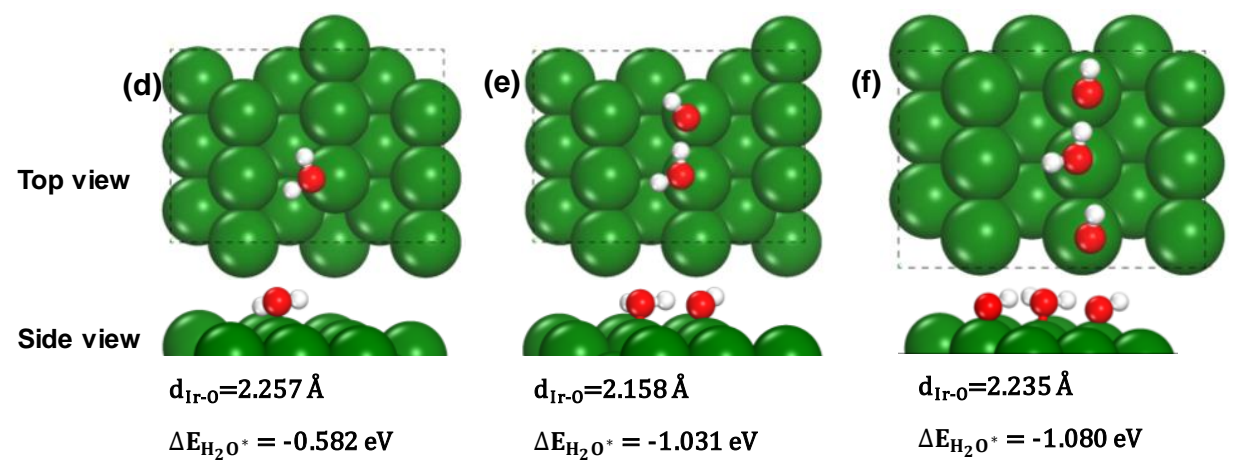

Figure S5 The stable adsorbed configurations of (a-c) $\mathrm{H}^{*}$ and $(\mathbf{d - f}) \mathrm{H}_{2} \mathrm{O}^{*}$ on $\operatorname{Ir}(110)$, green balls

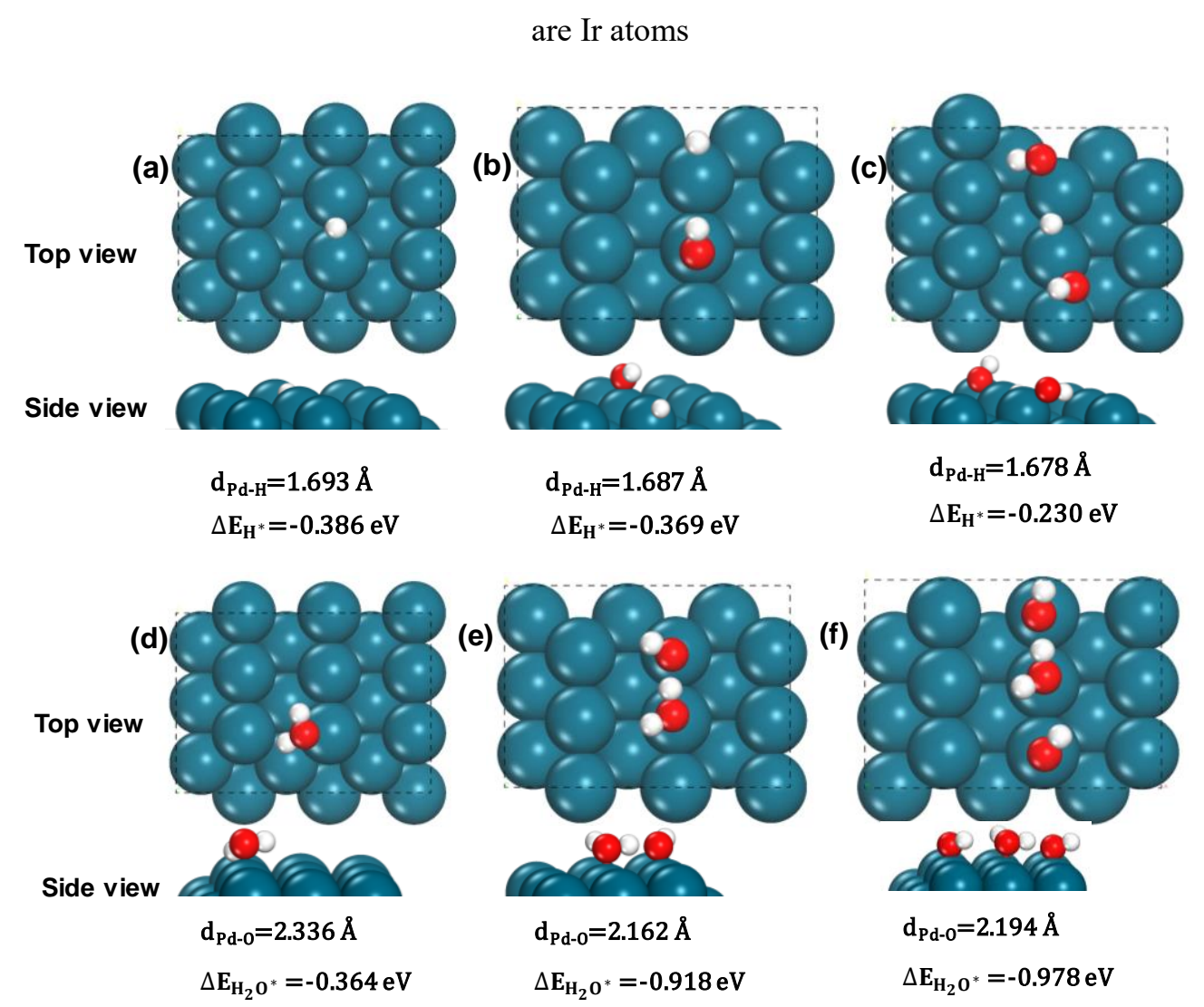

Figure S6 The stable adsorbed configurations of (a-c) $\mathrm{H}^{*}$ and (d-f) $\mathrm{H}_{2} \mathrm{O}^{*}$ on $\mathrm{Pd}(110)$, olive blue balls represent $\mathrm{Pd}$ atoms

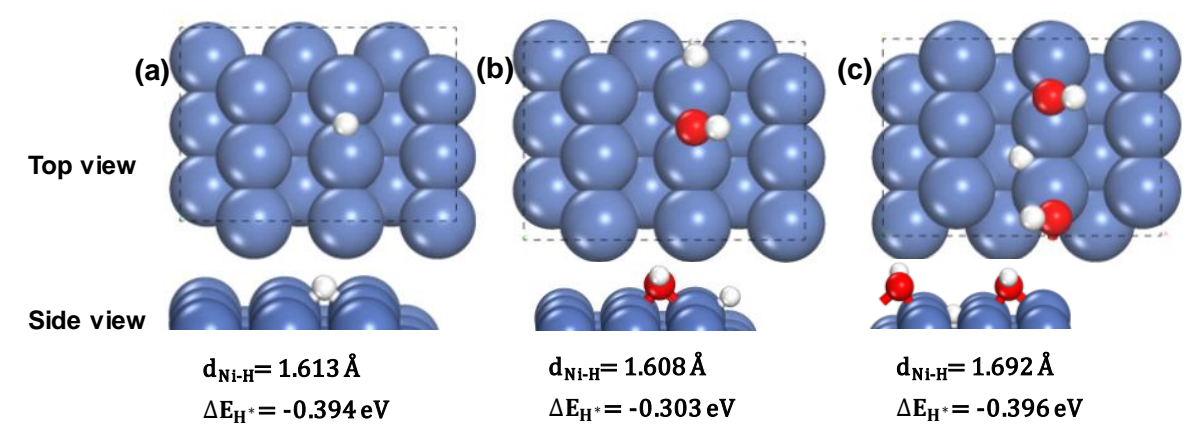




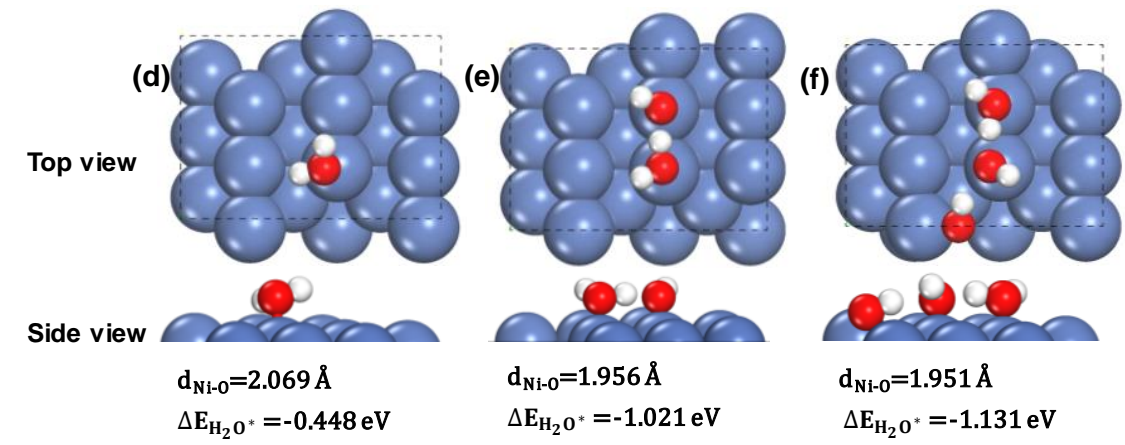

Figure S7 The stable adsorbed configurations of (a-c) $\mathrm{H}^{*}$ and (d-f) $\mathrm{H}_{2} \mathrm{O}^{*}$ on $\mathrm{Ni}(110)$, purple balls denote Ni atoms

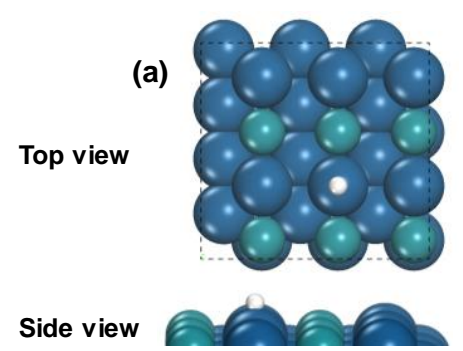

(b)
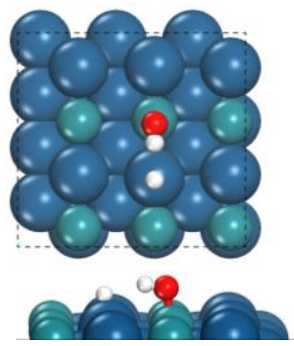

$\mathrm{d}_{\mathrm{Pt}-\mathrm{H}}=1.611 \AA$

$\Delta \mathbf{E}_{\mathbf{H}^{*}}=-0.255 \mathrm{eV}$

(e) (d)

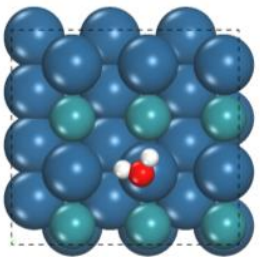

Side view

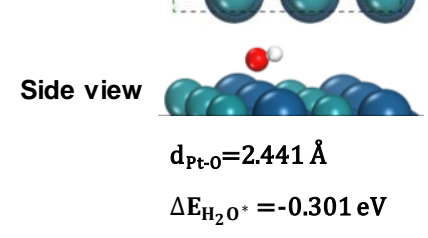$$
\Delta \mathbf{E}_{\mathrm{H}^{*}}=-0.162 \mathrm{eV}
$$

Top view
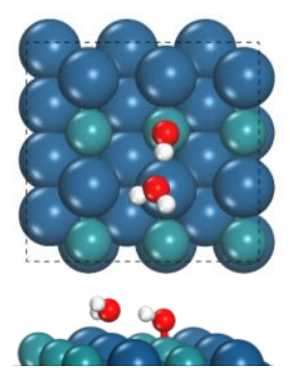

$\mathrm{d}_{\mathrm{Pt} .0}=2.540 \AA$

$\Delta \mathbf{E}_{\mathrm{H}_{2} \mathbf{0}^{*}}=-0.417 \mathrm{eV}$ (c)
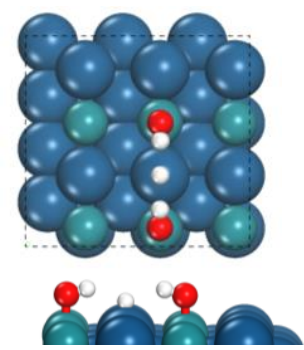

$\mathrm{d}_{\mathrm{Pt}-\mathrm{H}}=1.620 \AA$

$\Delta \mathbf{E}_{\mathrm{H}^{*}}=-0.247 \mathrm{eV}$

(f)
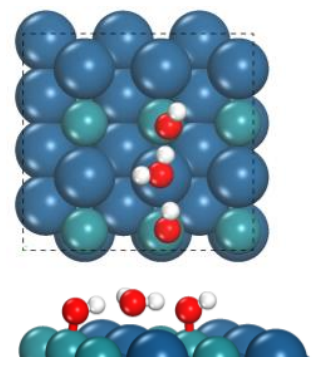

$\mathrm{d}_{\mathrm{Pt}-0}=2.340 \AA$

$\Delta \mathbf{E}_{\mathrm{H}_{2} \mathbf{0}^{*}}=-0.910 \mathrm{eV}$

Figure S8 The stable adsorbed configurations of $\mathrm{H}^{*}(\mathbf{a}-\mathbf{c})$ and $\mathrm{H}_{2} \mathrm{O}^{*}(\mathbf{d}-\mathbf{f})$ on $\mathrm{PtRu}(110)$, green balls represent $\mathrm{Ru}$ atoms 


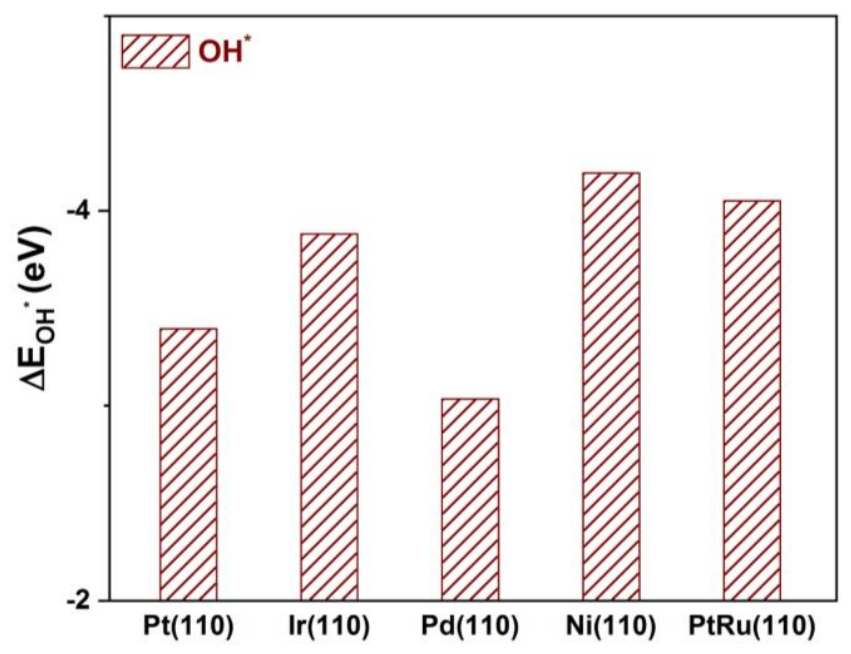

Figure $\mathbf{S 9} \mathrm{OH}^{*}$ adsorption energy on different metals
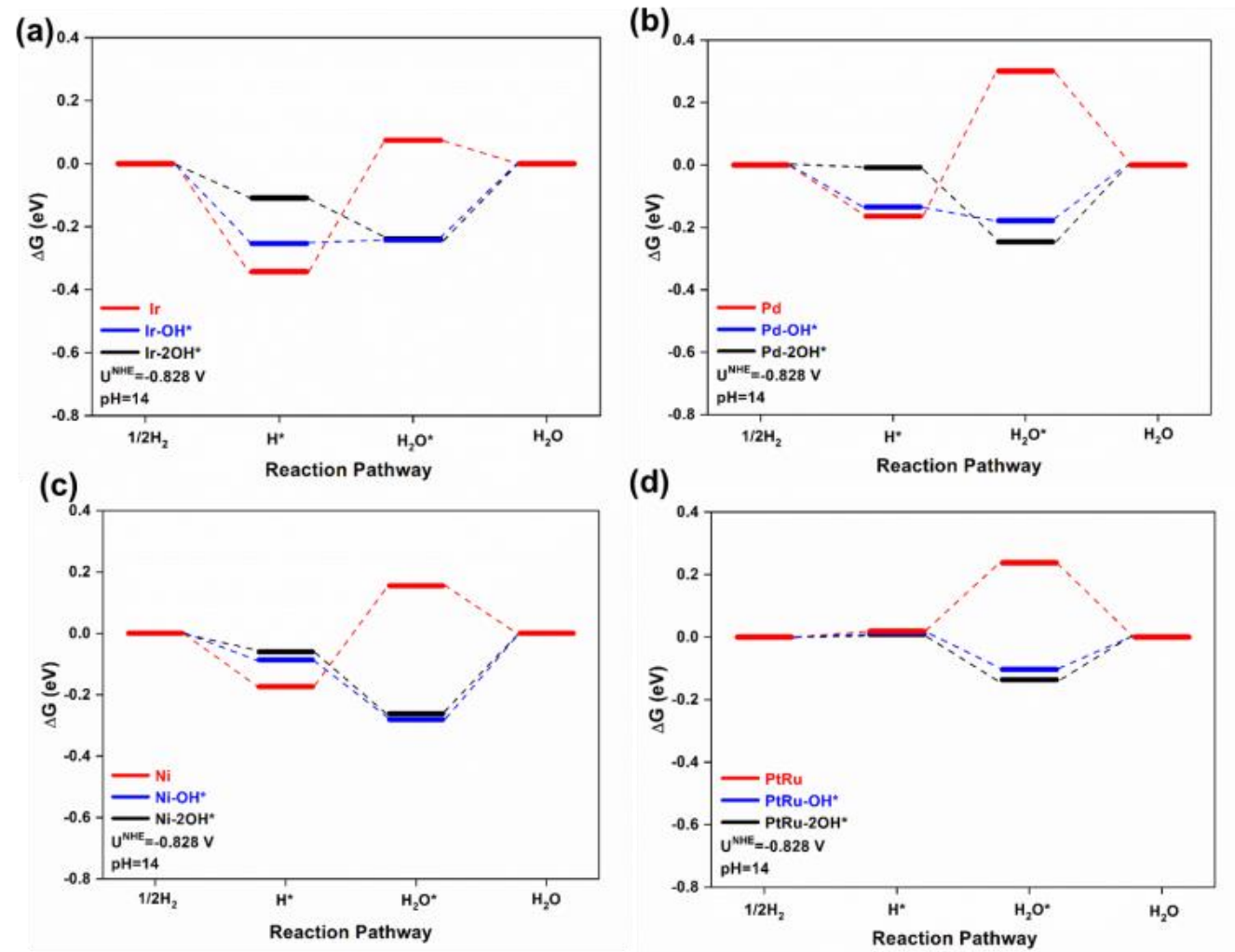

Figure S10 Gibbs free energy diagrams for HOR on (a) $\operatorname{Ir}(110)$, (c) $\operatorname{Pd}(110),(\mathbf{e}) \mathrm{Ni}(110)$ and (g) $\operatorname{PtRu}(110)$. 


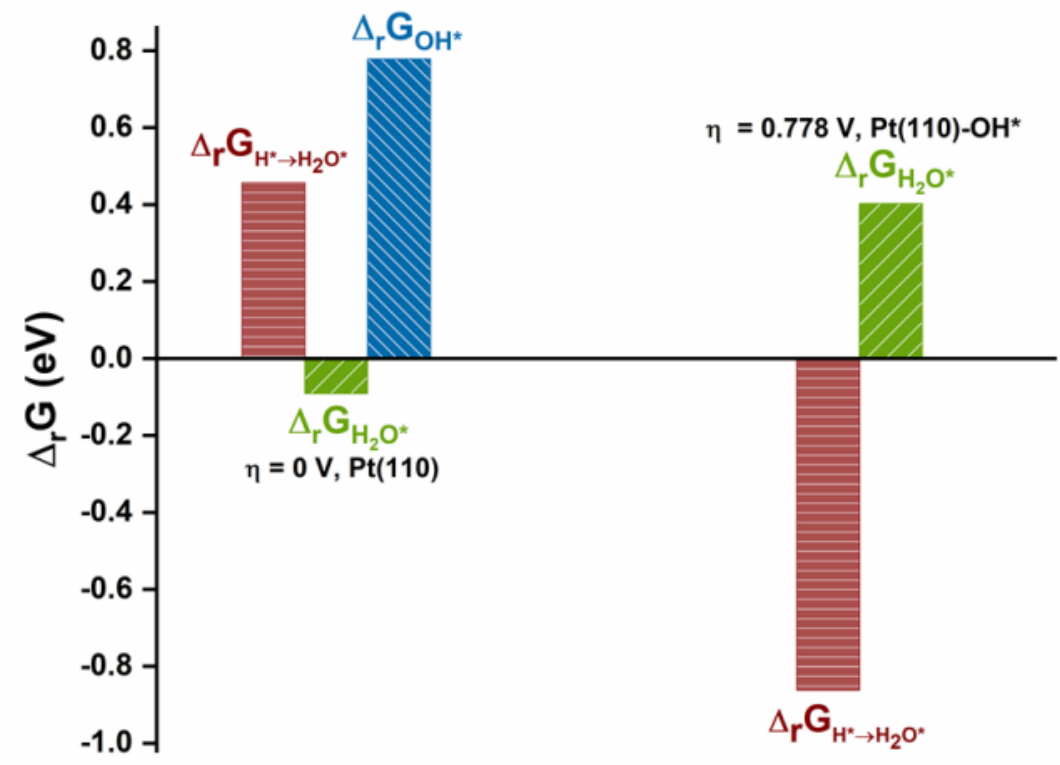

Figure S11 The $\Delta_{\mathrm{r}} \mathrm{G}_{\mathrm{H}^{*} \rightarrow \mathrm{H}_{2} \mathrm{O}^{*}}, \Delta_{\mathrm{r}} \mathrm{G}_{\mathrm{OH}^{*}}$ and $\Delta_{\mathrm{r}} \mathrm{G}_{\mathrm{H}_{2} \mathrm{O}^{*}}$ at overpotential $\eta(\mathrm{V})$ equals 0 or $\mathrm{U}_{\mathrm{OH}^{*}}+0.828 \mathrm{~V}$ on $\mathrm{Pt}(110)$

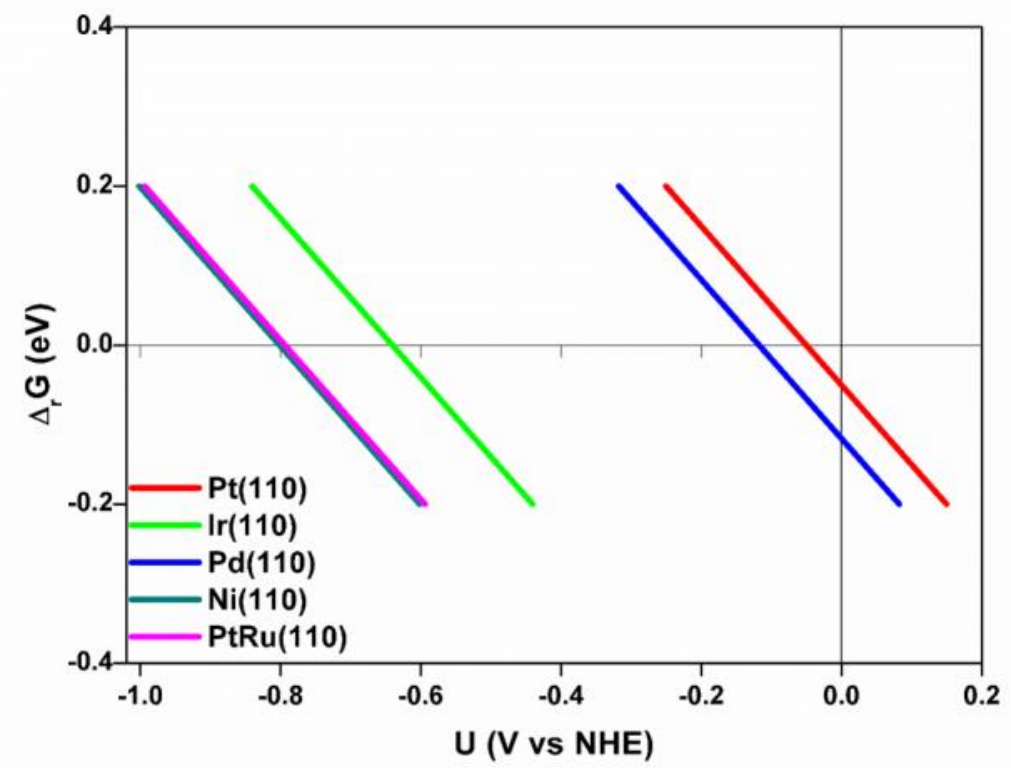

Figure S12 Gibbs free energy diagram for $\mathrm{OH}^{*}$ formation on $\mathrm{M}(110)(\mathrm{M}=\mathrm{Pt}, \mathrm{Ir}, \mathrm{Pd}, \mathrm{Ni}$ and $\mathrm{PtRu})$ at varied potentials (U, $\mathrm{V}$ vs NHE) 


\section{REFERENCES}

(1) Bajdich, M.; Garcia-Mota, M.; Vojvodic, A.; Norskov, J. K.; Bell, A. T. Theoretical Investigation of the Activity of Cobalt Oxides for the Electrochemical Oxidation of Water. J. Am. Chem. Soc. 2013, 135, 13521-13530.

(2) David R.Lide. Handbook of Chemistry and Physics, 8-25-8-30, 78th. edition, 1997-1998 